\title{
Possible Dark Matter annihilation in the galaxy cluster A520
}

\section{P. Marchegiani*}

School of Physics, University of the Witwatersrand, Private Bag 3, 2050-Johannesburg, South Africa

E-mail: Paolo.Marchegiani@wits.ac.za

\section{S. Colafrancesco ${ }^{\dagger}$}

School of Physics, University of the Witwatersrand, Private Bag 3, 2050-Johannesburg, South Africa

\section{N.F. Khanye}

School of Physics, University of the Witwatersrand, Private Bag 3, 2050-Johannesburg, South Africa

\begin{abstract}
Dark Matter annihilation is a possible source of relativistic electrons in galaxy clusters; these electrons, interacting with intra-cluster magnetic fields, can contribute to the diffuse radio emission observed in several clusters. We explore the possibility that Dark Matter annihilation can be at the origin of the radio emission in the cluster A520. We find that, if a radio emission of Dark Matter origin is present in this cluster, it can be observed in a region in the NE part of the cluster, whereas we expect that in most parts of the cluster the emission from ordinary matter should be dominant. High-energy measurements in X-rays and gamma rays don't appear to be suitable to discriminate between ordinary and Dark Matter origin for the models we have considered, because the corresponding non-thermal emissions predicted in this cluster are well below the sensitivity of present and forthcoming instruments.
\end{abstract}

High Energy Astrophysics in Southern Africa - HEASA2018

1-3 August, 2018

Parys, Free State, South Africa

\footnotetext{
*Speaker.

${ }^{\dagger}$ Deceased
} 


\section{Introduction}

Galaxy clusters are dominated by a component of Dark Matter (DM) of unknown nature. The annihilation or decay of DM particles has been recognized as a possible source of relativistic electrons and gamma rays in galaxy clusters [1,2]. However, other mechanisms originated by ordinary matter (baryonic or leptonic) can produce non-thermal emission in galaxy clusters [3]. In clusters where the DM density spatial distribution, as derived from gravitational lensing measures, is different from the Intra Cluster Medium (ICM) one, as derived from X-rays, like for example in the Bullet cluster [4], the detection of DM-induced signals can be easier in the cluster regions where the DM is supposed to be dominant $[5,6]$.

A520 is a cluster where $\mathrm{X}$-rays and gravitational lensing measures reveal a situation similar to the case of the Bullet cluster. In fact, in A520 the distribution of the DM density presents two main peaks located on the opposite sides of the cluster geometrical centre along the NE-SW direction [7]; the X-ray emission instead shows a main peak close to the cluster geometrical centre, and an extension in the SW part of the cluster, where a second peak, associated to the Brightest Cluster Galaxy (BCG), is located close to the edge of the cluster [8]. This situation has been interpreted as the effect of a merging event taking place approximately perpendicularly to the line of sight, and confirmed by the detection of a bow shock associated to the BCG that is moving outwards after crossing the ICM $[9,10]$.

A520 hosts a giant bright radio halo $[11,12]$ having an integrated spectral index value between 325 and $1400 \mathrm{MHz}$ of $\alpha \sim 1.12$, that does not present a systematic steepening towards the peripheral regions [13]. The radio maps show that the halo is divided in two main sub-halos in the $\mathrm{NE}$ and SW regions of the cluster; interestingly, this structure is analogue to the structure of the DM distribution in the cluster. This fact suggests the DM annihilation as a possible origin for the diffuse radio emission in A520.

In a recent paper [14] we have studied this possibility, using the observational constraints available in this cluster, and constraints on DM models as derived from previous studies in other clusters like Coma [15]. Here we summarize the results we have obtained.

\section{Radio emission in the whole cluster}

We first study the radio emission expected in the whole cluster using a baryonic model, where secondary electrons are produced by hadronic interactions between cosmic rays and thermal protons [16]. We don't consider the possible presence of a leptonic component; the effect of such a component would be to increase the contribution given from ordinary matter (e.g. [17]), making more difficult to distinguish the presence of the DM contribution.

We also consider two DM models, where electrons are produced by DM annihilation [1], considering the cases of a neutralino with mass $9 \mathrm{GeV}$ and annihilation final state $\tau^{+} \tau^{-}$, and mass $43 \mathrm{GeV}$ and annihilation final state $b \bar{b}$. We consider these cases because they are suggested by an analysis of the gamma ray excess in the Galactic centre [18] and have been considered in other studies of DM annihilation in galaxy clusters [15]; other neutralino annihilation channels, or decay channels of other kinds of particles, are in principle possible, but their study is beyond the goal of the present paper. 
For the hadronic model we use a thermal gas density profile $n_{t h}(r)$ as derived from X-ray measures [11], and for non-thermal protons we assume a density spatial profile proportional to the thermal one, a power-law energy spectrum $N_{p}(\gamma) \propto \gamma^{-s_{p}}$ with several values of the spectral index $s_{p}$, and parametrize their density normalization through the ratio between the cosmic rays and thermal pressures, $P_{C R} / P_{t h}$ [19], that from gamma ray upper limits in clusters is expected to have upper limits of the order of 2-6\% [20,21]. Since there are not estimates of the magnetic field in A520 available in literature, we assume a magnetic field similar to the one found in Coma, with a central value of $5 \mu \mathrm{G}$ and a radial profile proportional to $n_{t h}(r)^{1 / 2}$ [22]. In principle, the properties of the magnetic field in A520, that is a more disturbed cluster than Coma, can be different from the Coma case; however, a different magnetic field would impact in a similar way both on the synchrotron power and the energy losses of all the models we are considering, so we don't expect that a different magnetic field configuration can change heavily the ratio between the hadronic and the DM emission.

For the DM models, we use the normalizations, given by the product of the annihilation cross section, $\langle\sigma v\rangle$, and the substructures boosting factor, $\mathscr{B}$, as obtained from the fitting to the flux of the radio halo in the Coma cluster [15], that can be considered as upper limits. For the radial distribution of the DM particles, we assume a Navarro, Frenk \& White distribution [23], where the values of the central density and the scaling radius can be derived from the DM halo mass [24].

In left panel of Fig.1 we compare the radio flux calculated according to these models with the observed spectrum of the radio halo in A520 [13]. We find that the hadronic model provides a good fit to the data for a spectral index $s_{p}=2.2$ and a pressure ratio $P_{C R} / P_{t h}=2.6 \%$, that is compatible with the upper limits derived from stacked analysis of gamma ray emission in clusters. The DM emission results instead to be lower than the observed flux by almost one order of magnitude, suggesting that the bulk of radio emission in A520 can not be of DM origin.

It is anyway possible that the DM can give a contribution in some regions of the cluster, as suggested in the case of the Bullet cluster [5]. In the next Section we explore this possibility.

\section{Radio emission in the DM peak region}

To search for regions of the cluster where a DM-induced emission can be dominant, we examined the X-rays and gravitational lensing maps, and compared them with the radio ones.

Gravitational lensing measures [7] show the presence of two massive DM halos located respectively in the NE and SW parts of the radio halo: the SW one is located close to the BCG that is moving outwards producing the detected bow shock; it is therefore reasonable to think that the radio emission in this region is dominated by the electrons (primary or secondary) originated by this galaxy or accelerated by the associated bow shock. The NE DM halo is instead located in a region with a weak X-ray emission, but close to a peak of the radio emission, visible in the NVSS [25] map (see left panel of Fig.2). The radio data analysis has been done using the CASA package, and the X-ray imaging (using Chandra data ObsID: 528, with exposure of $9.47 \mathrm{ks}$ ) was done using ASTROPY and ds9 packages, and smoothed with a Gaussian kernel with $\sigma=2$ pixels.

A closer inspection of this region is shown in right panel of Fig.2, where the contours from NVSS and TGSS [26] radio surveys, and from the gravitational lensing analysis are overlapped to the optical ESO-DSS map. The radio peak at the centre of the image is visible in the TGSS 

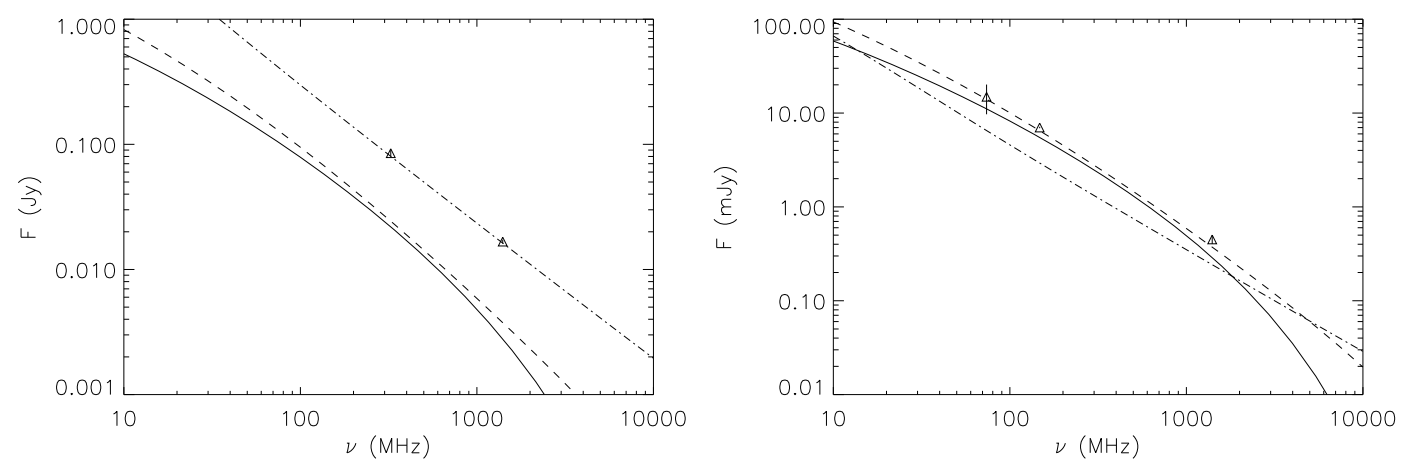

Figure 1: Left panel: spectrum of the radio halo flux in A520 for: $i$ ) a neutralino model with mass $M_{\chi}=9$ $\mathrm{GeV}$, annihilation final state $\tau^{+} \tau^{-}, \mathscr{B} \times\langle\sigma v\rangle=6 \times 10^{-25} \mathrm{~cm}^{3} \mathrm{~s}^{-1}$ (solid line); ii) a neutralino model with mass $M_{\chi}=43 \mathrm{GeV}$, annihilation final state $b \bar{b}, \mathscr{B} \times\langle\sigma v\rangle=4 \times 10^{-24} \mathrm{~cm}^{3} \mathrm{~s}^{-1}$ (dashed line); iii) a hadronic model with proton spectral index $s_{p}=2.2$ and a pressure ratio $P_{C R} / P_{t h}=2.6 \%$ (dot-dashed line); data are from Vacca et al. [13]. Right panel: spectrum of the radio halo flux in the NE DM region of A520 for a magnetic field of $5 \mu \mathrm{G}$ and for the same models than in the left panel, assuming a pressure ratio $P_{C R} / P_{t h}=4 \%$ for the hadronic model; data are from Table 1. Figures are from [14].
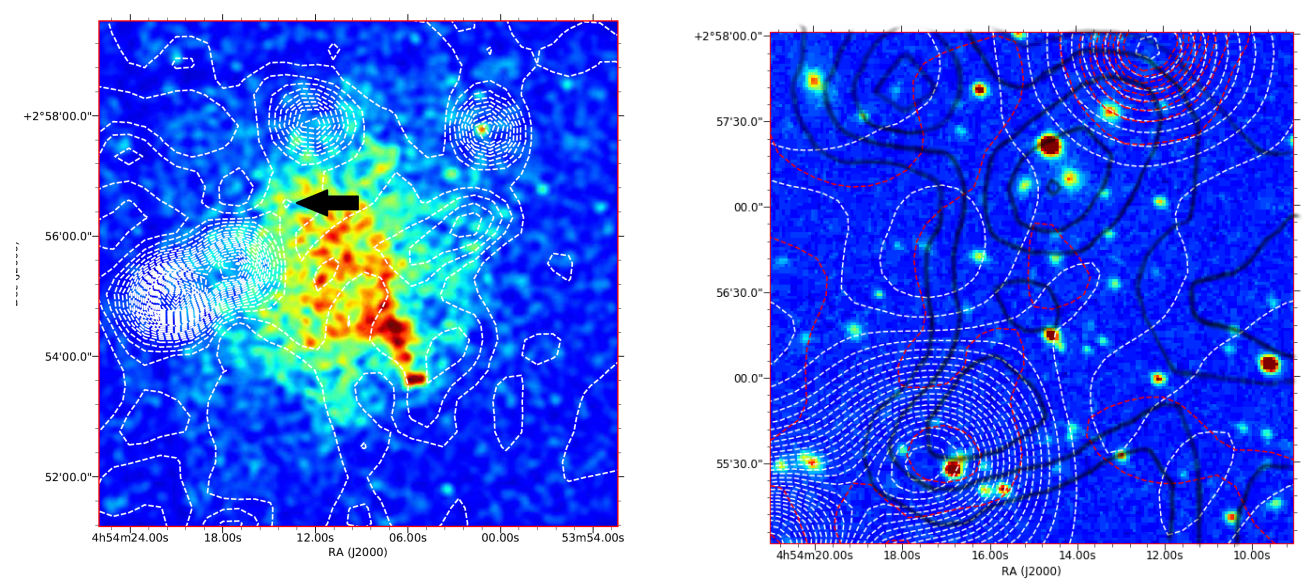

Figure 2: Left panel: smoothed X-ray image in the $0.8-4 \mathrm{keV}$ band of A520 from Chandra with NVSS contours overlapped (white); the black arrow indicates the peak of the NVSS map close to the NE DM halo. Right panel: optical image of the NE DM region from ESO DSS with contours from NVSS (white), TGSS (red), and weak lensing map [8] (black) overlapped. Figures are from [14].

contours, and is located between two lensing peaks; the distance between the centre of the TGSS peak and the lensing peaks is of the order of the TGSS resolution ( $25 \operatorname{arcsec})$, and therefore it is possible that in this peak there is a contribution from DM annihilation.

We estimated the radio flux coming from this region, in a circle centred at the coordinates $\mathrm{RA}=0454$ 15.0, Dec=+02 56 31.4, and with a radius of 35 arcsec (i.e. avoiding the emission from the two evident radio galaxies in the region), by analyzing publicly available data from radio surveys VLSSr [27], TGSS, and NVSS; the results are reported in Table 1. 


\begin{tabular}{ccc}
\hline$S_{74}(\mathrm{mJy})$ & $S_{150}(\mathrm{mJy})$ & $S_{1400}(\mathrm{mJy})$ \\
\hline $14.9 \pm 5.2$ & $7.01 \pm 0.13$ & $0.450 \pm 0.047$ \\
\hline
\end{tabular}

Table 1: Radio fluxes estimated in a circular region centred in $R A=0454$ 15.0, Dec=+02 56 31.4, and with a radius of $35 \mathrm{arcsec}$, as obtained from the surveys VLSSr (74 MHz), TGSS (150 MHz), and NVSS (1.4 $\mathrm{GHz})$.

Then we calculated the hadronic and the DM emission in this region for the same models described in the previous Section. Since the size of this region is small compared to the core radius of the cluster, we use a uniform magnetic field intensity and uniform densities of thermal gas and non-thermal protons inside the region. For the hadronic model we assume a reference value of pressure ratio $P_{C R} / P_{t h}=4 \%$, higher than the one providing the best fitting to the whole radio halo. For the DM model, we use the parameters of the density spatial distribution obtained for a halo with mass $M=4.08 \times 10^{13} \mathrm{M}_{\odot}$ [7]. We note that the derived core radius of the DM distribution for this mass is $\sim 112 \mathrm{kpc}$, of the order of the radius of the region from where we extracted the radio flux ( $\sim 118 \mathrm{kpc})$.

From right panel of Fig.1 we see that the DM emission in this region appears to be dominant with respect to the hadronic one. This is because in a small region the contribution of the central peak of the DM density is more important than the one provided by the flat density profile of nonthermal protons. We found that it is possible to obtain a radio flux produced by DM annihilation similar to the observed one for a magnetic field of the order of $5 \mu \mathrm{G}$. We note that the model with neutralino mass $43 \mathrm{GeV}$ reproduces the spectral shape of the radio emission of this region better than the $9 \mathrm{GeV}$ model.

Therefore, the DM emission in the NE region can be dominant on the hadronic one if the normalization of the DM emission is the same than the one that can reproduce the radio halo flux in Coma, and this suggests that the contribution of DM to the radio emission in the cluster, if present, can be found in this region.

\section{High energy emission}

In this Section we discuss if the non-thermal emission produced in the high energy bands (Xray and gamma ray) is suitable to be detected with present or forthcoming instruments, in order to understand if with this kind of observations it is possible to discriminate between the different models and derive more information on the properties and the origin of cosmic ray particles in A520 and in the NE region.

In Fig. 3 we show the high-energy non-thermal emission, given by the sum of the Inverse Compton Scattering of the CMB photons and non-thermal bremsstrahlung with the thermal ions from the secondary electrons, and the gamma ray emission produced by neutral pions decay, for the three models we used in the past Section, and for the two regions we considered: the whole cluster (left panel), and the NE region (right panel). In this Figure we don't show the thermal bremsstrahlung emission from the hot gas in the ICM, that is dominant in the soft X-ray band and is shown in the Chandra map (left panel of Fig.2).

In the whole cluster the hadronic emission dominates over the emission of DM origin along the whole spectrum, whereas in the NE region the DM emission is stronger than the hadronic one 

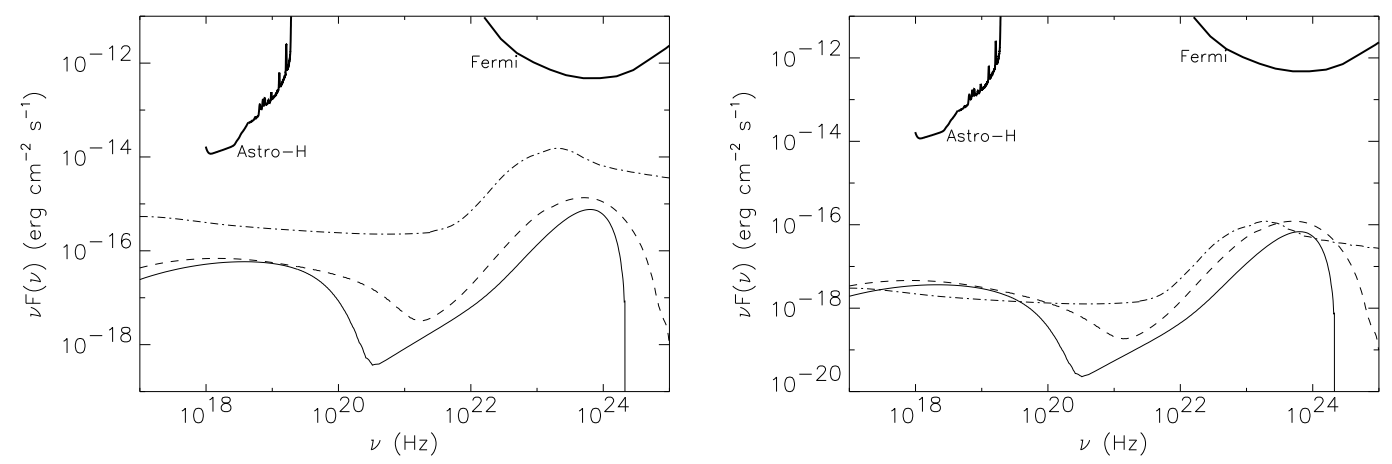

Figure 3: Spectral energy distribution of the high energy non-thermal emission from the whole cluster A520 (left panel) and the NE region (rigth panel) for: $i$ ) a neutralino model with mass $M_{\chi}=9 \mathrm{GeV}$, annihilation final state $\tau^{+} \tau^{-}, \mathscr{B} \times\langle\sigma v\rangle=6 \times 10^{-25} \mathrm{~cm}^{3} \mathrm{~s}^{-1}$ (solid line); ii) a neutralino model with mass $M_{\chi}=43$ $\mathrm{GeV}$, annihilation final state $b \bar{b}, \mathscr{B} \times\langle\sigma v\rangle=4 \times 10^{-24} \mathrm{~cm}^{3} \mathrm{~s}^{-1}$ (dashed line); iii) a hadronic model with proton spectral index $s_{p}=2.2$ and pressure ratios $P_{C R} / P_{t h}=2.6 \%$ in the whole cluster and $4 \%$ in the NE region (dot-dashed line). We also show the sensitivity curves of Astro-H for $100 \mathrm{ks}$ of time integration (from http://astro-h.isas.jaxa.jp/researchers/sim/sensitivity.html) and Fermi-LAT for 10 yrs [28]. Figures are from [14].

in the X-ray band (between 0.3 and $400 \mathrm{keV}$ for the $43 \mathrm{GeV}$ model, and between 1 and $150 \mathrm{keV}$ for the $9 \mathrm{GeV}$ model), while in the gamma rays the hadronic emission is generally stronger than the DM one, except for the spectral region between 1 and $10 \mathrm{GeV}$ for the $43 \mathrm{GeV}$ mass model, and between 2 and $4 \mathrm{GeV}$ for the $9 \mathrm{GeV}$ model. However, all these emissions are well below the sensitivity of present and forthcoming instruments (in the same Figure we show, for reference, the sensitivities of Astro-H for $100 \mathrm{ks}$ of integration and Fermi-LAT for $10 \mathrm{yrs}$ ) even for the whole cluster, so we don't expect that observations in these bands can help to discriminate the origin of the non-thermal emission in A520.

\section{Discussion and conclusions}

Like in the Bullet cluster [5], in A520 the emission of DM origin probably is not producing the whole radio halo, but can be dominant in some regions of the cluster. In the whole cluster the baryonic emission is expected to be dominant on the DM one.

In the NE part of the cluster we have identified a peak of the radio halo located very close to one of the peaks of the DM distribution. In this region the emission of DM origin is expected to dominate on the emission of baryonic origin, if the DM has properties similar to the ones providing the best fitting to the spectrum of the radio halo in Coma [15]. The DM models produce a radio flux similar to the one we have estimated from publicly available surveys data (see Table 1) for a magnetic field of the order of $5 \mu \mathrm{G}$. Therefore, if a contribution from DM annihilation to the radio halo is present in this cluster, it needs to be searched in this region.

We note that, while in the Coma cluster the DM model providing the best fitting to the radio halo spectrum is the one with mass $9 \mathrm{GeV}$ and annihilation final state $\tau^{+} \tau^{-}$, in the NE region of A520 the model providing the best fitting to the radio spectrum is the one with mass $43 \mathrm{GeV}$ and 
annihilation final state $b \bar{b}$. Therefore other studies, even considering similar situations in other clusters, need to be performed in order to clarify the strenght of this result.

We also found that the expected high energy non-thermal emission from the whole cluster and the NE region is well below the sensitivities of present and forthcoming instruments, and therefore measurements in high energy spectral bands do not appear to be suitable to obtain better information on the non-thermal emission in A520.

\section{Acknowledgements}

SC passed away during the preparation of this paper; he gave a valuable contribution to its realization and we strongly thank him. This work is based on the research supported by the South African Research Chairs Initiative of the Department of Science and Technology and National Research Foundation of South Africa (Grant No 77948). PM and NK acknowledge support from the Department of Science and Technology/National Research Foundation (DST/NRF) Square Kilometre Array (SKA) post-graduate bursary initiative under the same Grant. We thank the Referee for useful comments and suggestions.

\section{References}

[1] Colafrancesco S., Profumo S., Ullio P., 2006, A\&A, 455, 21

[2] Huang X., Vertongen G., Weniger C., 2012, JCAP, 01, 042

[3] Feretti L., Giovannini G., Govoni F., Murgia M., 2012, A\&ARv, 20, 54

[4] Clowe D., et al., 2006, ApJ, 648, L109

[5] Marchegiani P., Colafrancesco S., 2015, MNRAS, 452, 1328

[6] Marchegiani P., Colafrancesco S., 2017, Proceedings of the 5th Annual Conference on High Energy Astrophysics in Southern Africa, PoS(HEASA2017)006

[7] Clowe D., Markevitch M., Bradac M., Gonzalez A.H., Chung S.M., Massey R., Zaritsky D., 2012, ApJ, 758, 128

[8] Wang Q.H.S., Markevitch M., Giacintucci S., 2016, ApJ, 833, 99

[9] Markevitch M., Govoni F., Brunetti G., Jerius D., 2005, ApJ, 627, 733

[10] Wang Q.H.S., Giacintucci S., Markevitch M., 2018, ApJ, 856, 162

[11] Govoni F., Feretti L., Giovannini G., Böhringer H., Reiprich T.H., Murgia M., 2001, A\&A, 376, 803

[12] Govoni F., Markevitch M., Vikhlinin A., van Speybroeck L., Feretti L., Giovannini G., 2004, ApJ, 605,695

[13] Vacca V., Feretti L., Giovannini G., Govoni F., Murgia M., Perley R.A., Clarke T.E., 2014, A\&A, 561, A52

[14] Marchegiani P., Colafrancesco S., Khanye N.F., 2019, MNRAS, 483, 2795

[15] Marchegiani P., Colafrancesco S., 2016, JCAP, 11, 033

[16] Blasi P., Colafrancesco S., 1999, Astroparticle Physics, 12, 169 
[17] Zandanel F., Pfrommer C., Prada F., 2014, MNRAS, 438, 124

[18] Abazajian K.N., Keeley R.E., 2016, Phys. Rev. D, 93, 083514

[19] Marchegiani P., Perola G.C., Colafrancesco S., 2007, A\&A, 465, 41

[20] Huber B., Tchernin C., Eckert D., Farnier C., Manalaysay A., Straumann U., Walter R., 2013, A\&A, 560, A64

[21] Prokhorov D.A., Churazov E.M., 2014, A\&A, 567, A93

[22] Bonafede A., Feretti L., Murgia M., Govoni F., Giovannini G., Dallacasa D., Dolag K., Taylor G.B., 2010, A\&A, 513, A30

[23] Navarro J.F., Frenk C.S., White S.D.M., 1996, ApJ, 462, 563

[24] Bullock J.S., et al., 2001, MNRAS, 321, 559

[25] Condon J.J., Cotton W.D., Greisen E.W., Yin Q.F., Perley R.A., Taylor G.B., Broderick J.J., 1998, AJ, 115,1693

[26] Intema H.T., Jagannathan P., Mooley K.P., Frail D.A., 2017, A\&A, 598, A78

[27] Lane W.M., Cotton W.D., van Velzen S., Clarke T.E., Kassim N.E., Helmboldt J.F., Lazio T.J.W., Cohen A.S., 2014, MNRAS, 440, 327

[28] Funk S., Hinton J.A., 2013, Astroparticle Physics, 43, 348 